“ (C) 2018 IEEE. Personal use of this material is permitted. Permission from IEEE must be obtained for all other uses, in any current or future media, including

reprinting/republishing this material for advertising or promotional purposes, creating new collective works, for resale or redistribution to servers or lists, or reuse of any copyrighted component of this work in other works." 


\title{
Predicting the Behavior of Induction Machine Using Motor-CAD and MATLAB Packages
}

\author{
For a comparison purpose
}

\author{
Ahmad A. Salah and Youguang Guo \\ Faculty of Engineering \& IT \\ University of Technology Sydney \\ Sydney, NSW 2007, Australia \\ Ahmad.a.salah@student.uts.edu.au \\ Youguang.Guo-1@uts.edu.au
}

\author{
David G. Dorrell \\ Electrical, Electronic \& Computer Engineering \\ University of KwaZulu-Natal \\ Durban 4041, South Africa \\ Dorrelld@ukzn.ac.za
}

\begin{abstract}
Optimization design method of induction machines is developed by using Computer-Aided Design and these machines are the most suitable choice for various and complex industrial applications. For instance, the wound rotor induction machines have enjoyed a renascence as the generator in many commercial wind turbines. In this paper, both Motor-CAD and MATLAB packages are employed to predict the electromagnetic behavior of the induction machine during steady-state and transient-state. Finite element analysis of a three-phase, four-pole induction machine is carried by using Motor-CAD and MATLAB in order to complete the comparison. The graphical interfaces of Motor-CAD environment will be utilized to describe the machine geometry, winding harmonics, material properties, and air-gap flux. The predicted results are validated by the experiment. Power losses are calculated for the test machine, and then the results will be explained.
\end{abstract}

Keywords-electromagnetic design; induction machine; impedance matrix; Motor-CAD

\section{INTRODUCTION}

An induction machine topology offers a low cost solution for various applications. On the other hand, their power density and power factor are not desirable. Designers have worked very hard on improving such design choices as the winding configuration, the skewing factor, and the ratio of stator to rotor slots. Since these issues seem to have deep outcomes in terms of electromagnetic behavior, which might appear as unsatisfactory run-up performance $[1,2]$. If the electromagnetic behavior could be predicted very accurately, this will help to adjust the manufacturing process in order to obtain higher efficiency and reliability of induction machines, especially for those operating under formidable environment conditions. In addition, analyzing the electromagnetic circuit of the induction machine is necessary to identify faults, because the faults produce one or more indicative signs in most cases, such as increased losses, torque pulsation, and unbalanced air-gap voltages and line currents [3].

Finite element and analytical methods are commonly used to estimate the electromagnetic performance of the induction machine since these methods are particularly suitable in developing credible approximations in calculating the current density distribution [4-6]. Finite element analysis of the induction machine is provided accurately by Motor-CAD simulation software and in less time than other softwares. Motor-CAD is a commercial software which is a specialist in thermal and electromagnetic analyses for a wide range of machines, such as brushless permanent magnet (BPM) motors, outer rotor BPM motors, induction motors, and permanent magnet DC machines [7, 8]. Motor-CAD was developed in 1999 by Motor Design Ltd, UK, which is a specialist thermal design package for electrical machines[9]. Generally, the machine performance, losses, and temperatures for induction machine can be calculated in few minutes in Motor-CAD environment. Furthermore, it allows the designers to optimize their designs for energy efficiency and size and cost reduction, offering a link between the electromagnetic design and thermal analysis of motors. It allows quick and easy evaluation of different cooling options during the design process in both cases of the steady-state and transient thermal analysis of electric machines. The authors in [8] used Motor-CAD to prove that a copper rotor induction motor offers a low-cost alternative to a permanent magnet motor for electric and hybrid vehicle traction. Analytical lumped-circuit technique and finite element analysis are used to predict the temperature rises of two identical prototype surface-mounted permanent magnet (PM) machines, and the results are compared with the measurement in [10]. The project in [11] has been designed for an externalrotor torque dense brushless PM motor, and this was done in Motor-CAD environment.

This paper will investigate how to design a three-phase, four-pole induction machine handled by Motor-CAD, and then modeling the electromagnetic performance of the machine. On the other hand, the impedance matrix technique including all coupling and mutual impedances in $[4,12]$ will be used in modeling and analysis of the same induction machine. It is not easy to obtain this impedance matrix using hand calculations, so it is reasonable to use MATLAB for calculations. The results of matrix method are verified using an invertedgeometry wound motor after programming it in MATLAB. The machine will be simulated in MATLAB $\backslash$ Simulink in order to present the machine losses, and these are repeated experimentally for the machine. The results (torque, current characteristic, power losses) are compared for the test machine. 
This paper is organized as follows: the machine geometry approach is reported in the second section of the paper. The third section is the implementation of an induction machine in Motor-CAD software which includes the electromagnet model for the test machine and the steady-state equivalent. In the fourth section, implementation of an induction machine using MATLAB is investigated in detail. Comparison work is reported in the fifth section including the loss calculation.

\section{MACHINE GEOMETRY}

In this paper, a three-phase, four-pole induction machine is investigated. Basic machine specifications are listed in TABLE I; some data were obtained from simulating the machine in CD-Adapco SPEED software. Geometry of the machine is set to 48 stator slots, 72 rotor slots, and 4 poles. Obviously, it is an old machine, in which the stator was rewound with a double layer winding and one parallel connection. This design aims to achieve the best possible winding in terms of MMF and its harmonics [13]. In the stator, the number of coils per phase equals 16, and the number of coils per pole per phase equals 4 (two coil sides per slot) as shown in Fig. 1. Concentrated winding was used in this machine. Also, there are 16 slots occupied by one phase in each layer, 8 with inward and 8 with outward current directions. With each layer each phase has to produce four poles in this case. The induction machine is shown in Fig. 2.

TABLE I. MACHINE SPECIFICATION

\begin{tabular}{|l|l|}
\hline \multicolumn{2}{|c|}{ Name plate details } \\
\hline Power [HP] & 10 (or 7.46 kW) \\
\hline Speed [rpm] & 1420 \\
\hline Frequency [Hz] & 50 \\
\hline Stator voltage [V] & $400 / 440$ Delta \\
\hline Stator rated current [A] & 13 \\
\hline Rotor voltage [V] & 200 \\
\hline Rated rotor current [A] & 22 \\
\hline Number of poles & 4 \\
\hline Slip [p.u.] & 0.0533 \\
\hline \multicolumn{2}{|c|}{ Measured geometry [mm] } \\
\hline Axial length of rotor core & 103.9 \\
\hline Axial length of stator core & 100.9 \\
\hline Stator outer diameter & 353 \\
\hline Stator inner diameter & 228.15 \\
\hline Rotor outer diameter & 226.42 \\
\hline Shaft diameter & 120 \\
\hline Airgap length & 0.5 \\
\hline Stator slots & 48 \\
\hline Stator slot opening & 3.9 \\
\hline Stator wire diameter & 1.534 \\
\hline Stator winding & Double layer lap with 12 slot pitch \\
\hline Coils per pole per phase & 4 \\
\hline Stator slot depth & 26.82 \\
\hline Stator tooth width & 8.22 \\
\hline Rotor slot opening & 3 \\
\hline Rotor slot depth & 22.5 \\
\hline Slot width & 3.5 \\
\hline Rotor wire diameter & $1.9-$ by calculation from SPEED \\
\hline Turns per coil & 17 in series \\
\hline Coils per phase & 16 \\
\hline & 2.1 \\
\hline Rph stator (DC, room temp.) & 0.23 \\
\hline Rph rotor (DC, room temp.) & \\
\hline
\end{tabular}

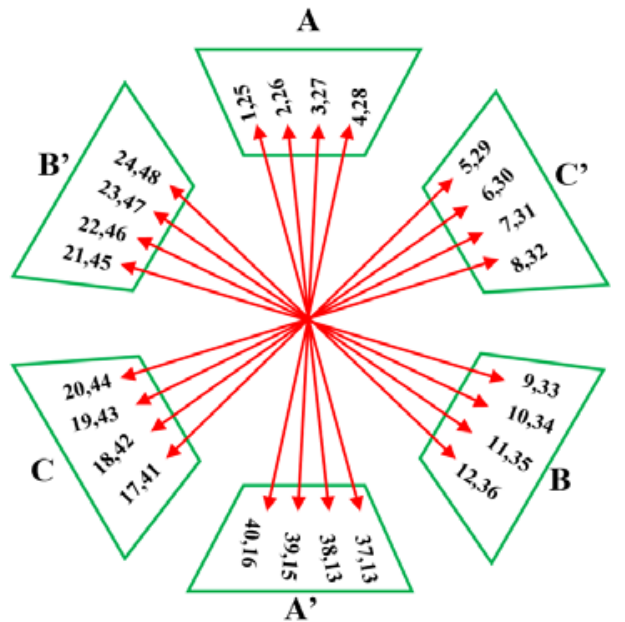

Fig. 1. The star of slot emf phasors for a double-layer winding (one layer shown) with 4 poles, $\mathrm{q}=4$ slots/pole/phase, Ns $=48$.

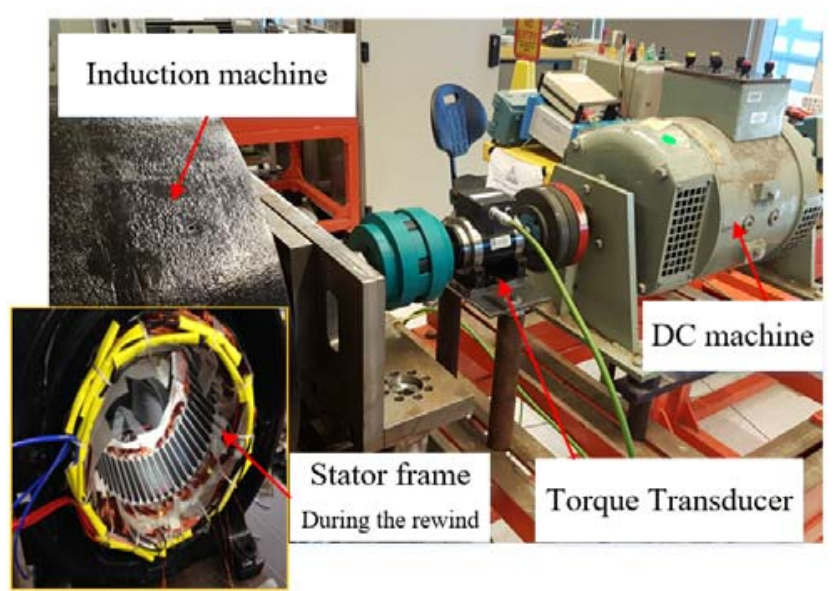

Fig. 2. Test machine, showing the stator frame during the rewinding process.

\section{IMPLEMENTATION OF AN INDUCTION MACHINE IN MOTOR- CAD}

Motor-CAD software has been introduced to provide a fast method for analyzing design changes on the behavior of the machine. Thus the designer can identify the variables such as geometry of the machine, material, and cooling type, which have the most influence on the machine performance. The numerical method (Finite Element Analysis) has been used in Motor-CAD to solve the electromagnetic circuit in the machines over the full torque/speed. The main advantage of this method is that it gives higher accuracy level in the results than the analytical methods. Using an intelligent loss algorithms make it suitable to many applications, for example, traction applications which have complex duty cycle loads.

It has very simple user-interface, so the designer just needs to identify the geometry of the machine and sets up a few winding, drive and material options and then all the difficult magnetic and heat transfer parameters can be calculated automatically in a couple of minutes. The machine geometry for the three-phase four-pole induction machine was set up, and the final figuration is shown in Fig. 3. 
The next step is to define the control parameters, so the software can calculate the machine performance. It allows running the machine as motor or generator to evaluate the electromagnetic performance. In addition, skew angle can be set which is utilized to reduce the machine harmonics. Then the winding pattern of the machine will be adjusted according to the initial geometry approach. Modifying the pattern for the slot/pole combination is available in Motor-CAD. The winding factors, phasor diagrams and winding harmonics for the test machine are shown in

Fig. 4. These charts in generally can provide an accurate comparison between different winding approaches, such as single layer and double layer windings. The peak harmonics amplitudes are reduced when the double layer is used instead of the single layer in the machine. A large materials database provides the commonly used materials in electric machines manufacturing. Furthermore, it has the capability to add other materials. Selecting cooling type is available and all difficult heat transfer data are calculated automatically. Motor-CAD certainly provides an exhaustive understanding of the cooling system and facilitates the optimization.

Electromagnetic model for the induction machine is calculated and the results of the analysis are depicted in Fig. 5. Air-gap of the machine is split into two symmetrical sections to represent stator air-gap and rotor air-gap during meshing while the electromagnetic field modeling is applied. Mesh points and boundary of the combining site are exactly the same for the coupling calculation of stator and rotor. In each time step, equations of magnetic field and electric field are calculated together for the iterative calculation. The matrix counter is refreshed cyclically for the next time step. The flux distribution in rotor and stator of the machine can be analyzed collectively or individually. Shading region options support investigating any flux region in the machine, for example, flux distraction on the stator or rotor surfaces.

The test machine was simulated over a $12 \mathrm{sec}$ period with 1 sec time interval to investigate the electromagnetic model of the machine at $400 \mathrm{~V}$ and $5 \mathrm{~A}$. Also locked rotor and open rotor tests were conducted. The supply current and terminal voltage are presented graphically in Motor-CAD. The back EMFs are calculated and then these waves can be customized by the back EMF graph options. All these components are taken into account when calculating equivalent circuit parameters of the induction machine after the solving is finished. Furthermore, the core loss resistance will be included in the equivalent circuit as in Fig. 6. One more feature for the software is that import/export geometry can also be done in the software environment. Fig. 7 shows the flux density in the airgap of the test machine with saturated and unsaturated conditions, providing more details about modeling of the magnetic circuit saturation. The magnetization curve of the machine material can be obtained. The out power, power factor, and various machine inductances are calculated. The power factor of the test machine was predicted to be low because it is an old machine which means that the stator and rotor resistance are slightly larger than the normal case. The designer can compare the different approaches of designing, which show the influence of the geometry of the machine as model assumptions. The models allow the designers to observe the transient processes. Self and mutual inductances are calculated for the same current values and the magnetizing and stator leakage inductances are extracted as in Fig. 7.

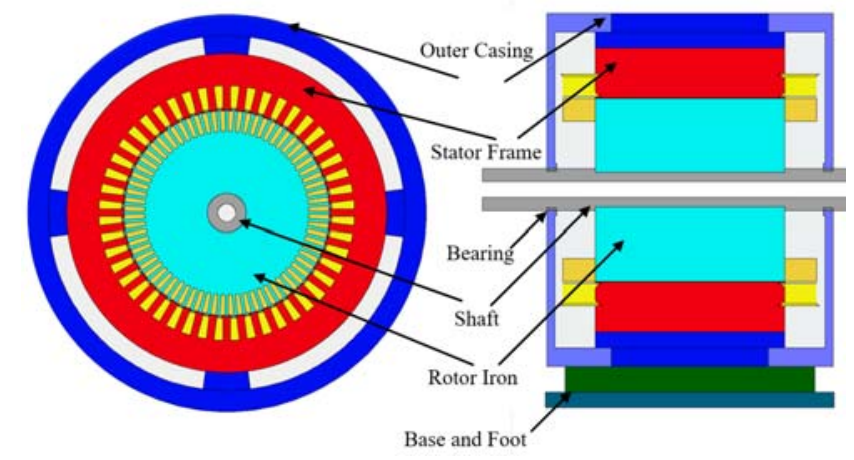

Fig. 3. Radial and axial sections of the test machine.

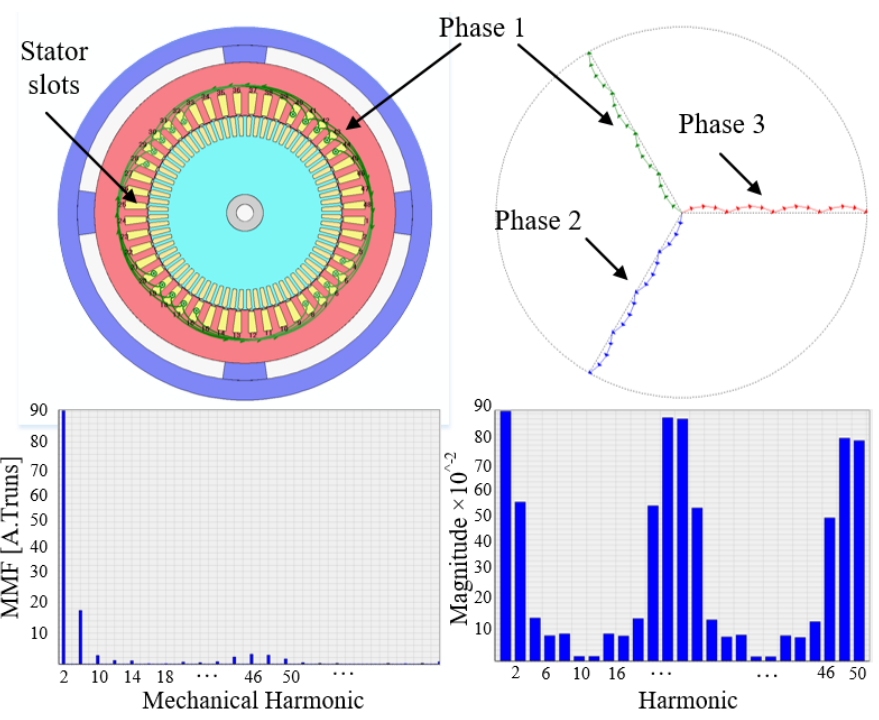

Fig. 4. Illustration of the winding layout (only phase 1 shown), phase diagram, winding factor, and the MMF harmonic of the test machine.

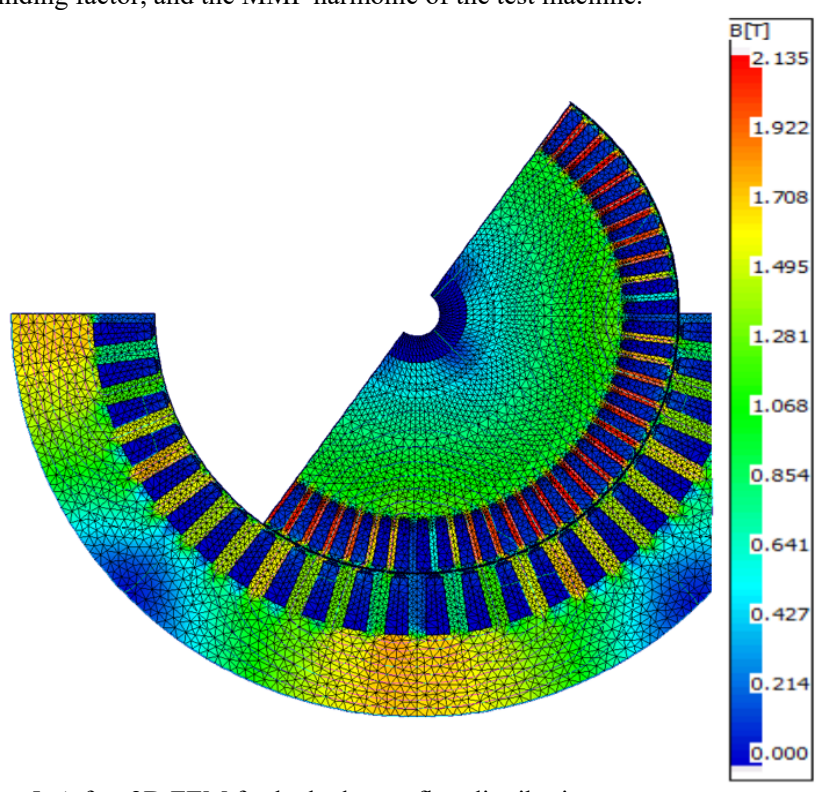

Fig. 5. A fast 2D FEM for locked rotor flux distribution. 


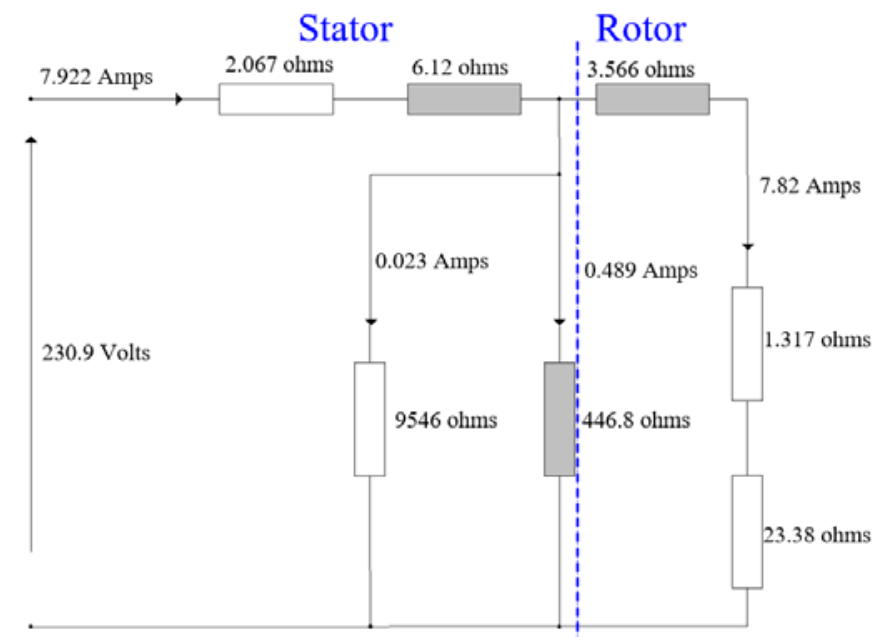

Fig. 6. The equivalent circuit of the test machine in Motor-CAD.
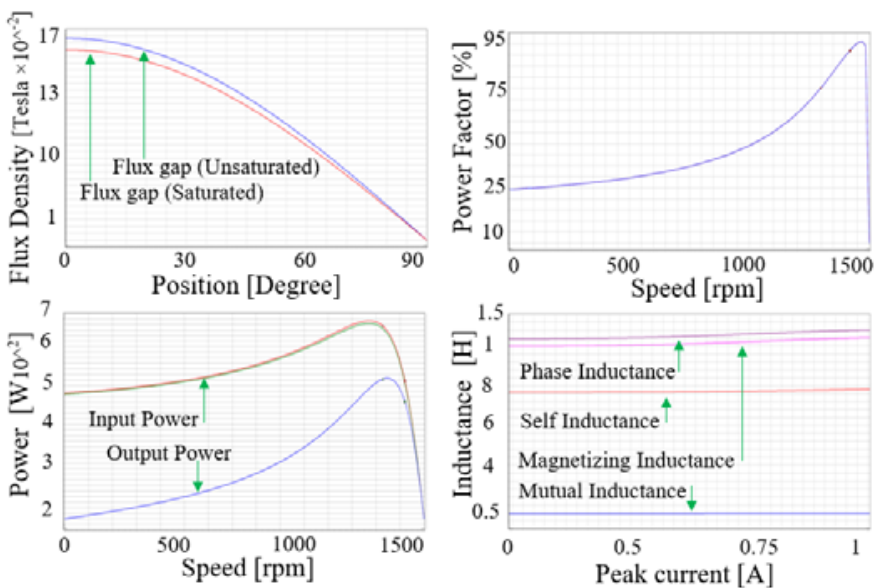

Fig. 7. Graphical results of the electromagnetic model in Motor-CAD.

\section{IMPLEMENTATION OF AN INDUCTION MACHINE IN MATLAB}

MATLAB is increasingly used in modeling and simulation of the electrical machines. Furthermore, MATLAB/Simulink implementation for an induction machine allows the designer to test various control strategies. The equations that describe the electromagnetic behavior of induction machine, which is based on rotating-field theory, can be coded in MATLAB. This paper presents a relatively simple but powerful model that can accurately analyze the behavior of induction machine using MATLAB. The model was coded in the form of a matrix which was applied for the test machine. This matrix technique described in [4] is used to find the steady state equivalent circuit for the wound rotor induction machine. The currents in the stator and rotor windings are linked to the supply voltage by means of the matrix equation as described in (1). The impedance matrix includes all mutual and coupling impedances with any distribution and number of rotor bars/phases investigated in [4], which are functions of the three-phase windings on the stator and rotor as in (1). The impedance matrix was developed to enhance the condition monitoring of the induction machine. The matrix depends on driving an expression for the conductor density distribution in terms of harmonic series; this can be done by using complex Fourier analysis.

$$
\left[\begin{array}{c}
\bar{v}_{s} \\
0 \\
\vdots \\
0
\end{array}\right]=\left[\begin{array}{cccc}
\bar{Z}_{s, s}^{n} & \bar{Z}_{s, 1}^{n} & \cdots & \bar{Z}_{s, N_{b}}^{n} \\
\bar{Z}_{1, s}^{n} & \bar{Z}_{1,1}^{n} & \cdots & \bar{Z}_{1, N_{b}}^{n} \\
\vdots & \vdots & \vdots & \vdots \\
\bar{Z}_{N_{b}, s}^{n} & \bar{Z}_{N_{b}, 1}^{n} & \cdots & \bar{Z}_{N_{b}, N_{b}}^{n}
\end{array}\right]\left[\begin{array}{c}
\bar{I}_{s} \\
\bar{I}_{1} \\
\vdots \\
\bar{I}_{N_{b}}
\end{array}\right]
$$

The matrix equation in (1) assumes that the rotor bars/phases are short-circuited. $N_{b}$ is the total number of the bars/phase windings in rotor, and $v_{s}$ is the main supply voltage. $I_{s}$ represents the stator current, and rotor bars currents labeled $I_{1}$, $I_{2}, \ldots$, and $I_{N b}$. The system of $N_{b}$ complex linear equations, where $N_{b}$ is the number of rows in the matrix, can be solved by any convenient means to find the $N_{b}$ unknown currents. $Z_{s, s}$ is the stator-stator coupling impedance, $Z_{1,1}$ and the first rotorrotor bar/phase coupling impedance. $Z s,_{1}$ and $Z_{1}, S$ are the rotorstator coupling impedance (for the first rotor bar/phase) and the stator-rotor coupling impedance (for the first rotor bar/phase) respectively. The rotor currents in (1) can be obtained from the knowledge of the applied voltages when the matrix is solved. As mentioned early, this technique was described in detail, and all the derivations of the mutual and coupling impedance can be found in [4].

The impedance matrix would be assembled and then the input/output power may be calculated in a straightforward manner using the stator voltage and current since the stator supply has been assumed to be ideal as in (2).

$$
P_{i}=\operatorname{Re}\left\{3 \bar{V}_{s} I_{s}^{*}\right\}
$$

The losses in the rotor or stator can be calculated from $I^{2} R$ losses in the rotor cage or stator winding and hence the mechanical output power for each harmonic and torque can also be calculated. The electromagnetic torque of induction machine is generated as a result of the interaction between the stator and rotor fields. Assume that the stator and rotor currents densities are $J_{s}(y, t)$ and $J_{r}(y, t)$ respectively. The developed torque is given by (3).

$$
T=\frac{2 \pi r^{2} \mu_{o} L_{s t}}{p_{m} g} \operatorname{Re}\left\{j\left(\bar{J}_{s} \bar{J}_{r}^{*}\right)\right\}
$$

The fundamental pole-pair number of the machine is $p_{m}$, the $L_{s t}$ is axial length of the stator, $g$ is air-gap length, and $r$ is average air-gap radius. The stator and rotor current densities are derived in [4]. The open circuit (running with light load) and the short circuit (locked rotor test) were carried out and the motor parameters were specified. The windings coefficient turn ratio was 3.78 , and this value is very close to the measured one of 3.56. The magnetizing reactance and core losses were calculated. The MATLAB Simulink was also performed for testing the machine losses and rotor current.

This algorithm is easily implementable in MATLAB software package and performs the most modern form of induction machine analytical analysis. 


\section{COMPARISON OF RESULTS}

A three-phase four-pole induction machine was used for implementing the work in this paper as shown in Fig. 1, and the data for the machine were presented in Table 1. The test of an induction machine is usually done by a test rig. This will run the machine up to synchronous speed, supplying the terminal voltage and loading the machine in a controlled manner. The speed will be reduced to standstill, and then the machine may be run to synchronous speed again. During these processes, the parameters such as stator current, power, power factor and torque can be recorded. To limit transient effects in the recording, the reading needs to be taken in the first few seconds only.

The developed torque and rotor current are shown in Figs. 8 and 9, which allow to compare the measured values and simulation values with ignorable harmonics components. It is sufficiently close to indicate that Motor-CAD and MALAB can accurately and fluently predict the behavior of machine induction machine. The similar values of the current induced in the rotor windings appear in Fig. 9. Again the increase in machine losses is related to the stator and rotor resistances which are considered high compared with the modern induction machine, which appears in Fig. 10. As would be expected losses are in good agreement, the differences being attributed to tolerances in the permanent magnet properties of the chosen construction materials.

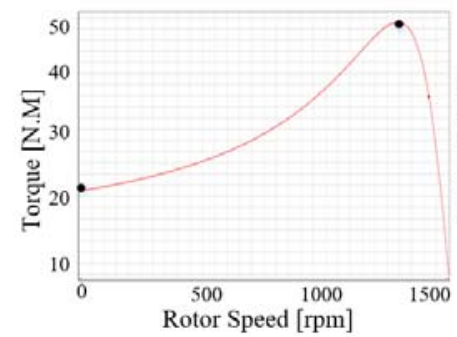

(a)

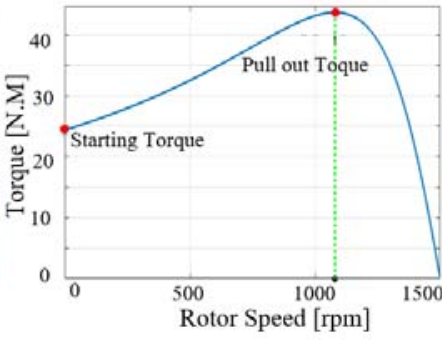

(b)
Fig. 8. Electromagnetic torque developed by the test machine v.s. speed characteristics: a) in Motor-CAD, b) in MATLAB.

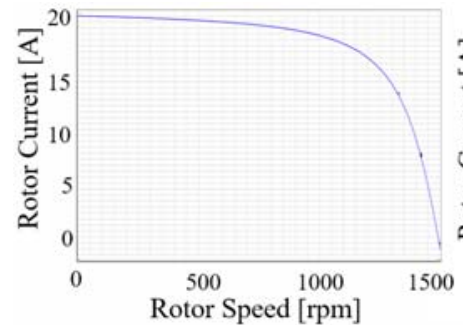

(a)

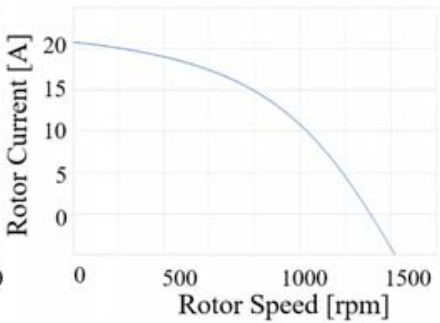

(b)
Fig. 9. Simulated rotor currents v.s. speed characteristics: a) in Motor-CAD, b) in MATLAB.

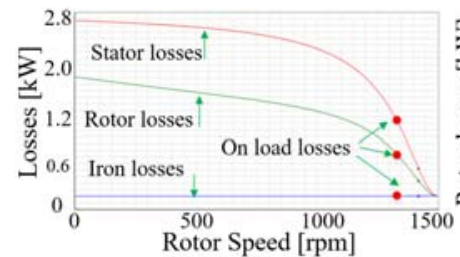

(a)

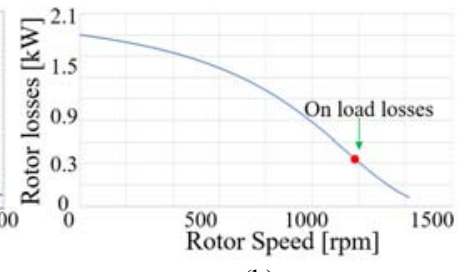

(b)
Fig. 10. Simulated losses v.s. speed characteristics: a) in Motor-CAD, b) in MATLAB.

\section{CONCLUSION}

Simulation models of induction machine using Motor-CAD and MATLAB packages are presented in this paper in detail. These models can certainly predict and characterize the behavior of the induction machine. Obviously the main purpose is to improve the design approaches, and at the end to improve the efficacy and reliability of induction machine. A comparison has been done between two models split and with the corresponding experimental work. MATLAB has the capacity to predict and evaluate induction machine behavior, especially for education purpose. However, it is a slightly complicated software and the designers often prefer simple software. On the other hand, Motor-CAD focuses only on electric motor design including induction machine. It provides very simple user-interface which simplifies the machine design consultancy \& training and involves visibly in large research projects. Motor-CAD EMag, Therm and Lab modules are developed to introduce fast and accurate analysis in one integrated software. Moreover, smart loss calculation algorithms which are coded in assembly language were included to speed up solving. The Correction factors are available within the Motor-CAD that are known from literature or from experimental data, and this make the results very accurate.

The FEA thermal solver in Motor-CAD enables fast calculation of the winding thermal resistance network and enhanced visualization of the winding hotspot location. It was shown that the combined electromagnetic and thermal analysis of an induction machine would be involved in the design procedure to account the correct thermal system and correct rate of the cooling.

A combination of analytical and numerical modeling is needed in modern practice. Motor-CAD has been designed to optimize the models that will numerically simulate the electrical machine and to support models that analytically simulate the external circuits such as inverters, connections, and gearboxes. This will achieve the balance, and push the electric machine industry forward.

The study on thermal systems of three-phase induction machine using Motor-CAD will be carried out in the future work.

\section{ACKNOWLEDGMENT}

The authors would like to express their sincere thanks and appreciation to Motor Design Ltd (MDL) for their support given through a free Motor-CAD license to complete the project. 


\section{REFERENCES}

[1] I. Boldea, The Induction Machines Design Handbook. CRC Press, 2009.

[2] R. Krishnan, Switched Reluctance Motor Drives: Modeling, Simulation, Analysis, Design, and Applications. CRC Press, 2001.

[3] A. Salah, Y. Guo, and D. Dorrell, " Monitoring and damping unbalanced magnetic pull due to eccentricity fault in induction machines: A review,' in Electrical Machines and Systems (ICEMS), 2017 20th IEEE Inter. Conf. on , 2017, pp. 1-6.

[4] A. Salah, Y. Guo, and D. Dorrell, " Impedance matrix analysis technique in wound rotor induction machines including general rotor asymmetry," 42nd Annual conf. of the IEEE Ind. Electronics Society, IECON 2, 2016, pp. 1821-1826.

[5] D. G. Dorrell, W. T. Thomson, and S. Roach, "Analysis of airgap flux, current, and vibration signals as a function of the combination of static and dynamic airgap eccentricity in 3-phase induction motors," IEEE Trans. Ind. Applications, vol. 33, no. 1, pp. 24-34, 1997.

[6] D. G. Dorrell, "Calculation of unbalanced magnetic pull in cage induction machines," Thesis, University of Cambridge, 1993.

[7] K. Shahzad, Y. Guo, L. Li, and D. Dorrell, "Design of high speed permanent magnet generator for solar co-generation system using motor-
CAD," IEEE Electrical Machines and Systems (ICEMS), 2017 20th Intern. Conf., 2017, pp. 1-6.

[8] J. Goss, M. Popescu, and D. Staton, "A comparison of an interior permanent magnet and copper rotor induction motor in a hybrid electric vehicle application," Electric Machines \& Drives Conf. (IEMDC), 2013 IEEE Inter., 2013, pp. 220-225.

[9] D. Dorrell, D. Staton, and M. McGilp, "A combined electromagnetic and thermal approach to the design of electrical machines," IEEE IECON Conf. 2006, 2006.

[10] N. Zhao, Z. Zhu, and W. Liu, "Thermal analysis and comparison of permanent magnet motor and generator," IEEE Electrical Machines and Systems (ICEMS), 2011 Inter. Conf., 2011, pp. 1-5.

[11] R. Wrobel, N. McNeill, D. Staton, J. Booker, and P. Mellor, "Torque dense, external rotor hub-drive for a hybrid solar vehicle," IEEE Vehicle Power and Propulsion Conf. VPPC, 2006.

[12] D. G. Dorrell, "Matrix analysis techniques in cage induction machines," Inter. Journal of Computer Appl. in Tech., vol. 36, no. 2, pp. 69-76, 2009.

[13] J. Pyrhonen, T. Jokinen, and V. Hrabovcova, Design of rotating electrical machines. John Wiley \& Sons, 2013. 